\title{
Session 5: Autoimmunity
}

\author{
Wednesday 6 October 2004. Moderator: Yehuda Shoenfeld
}

[16.45-17.30]

[Keynote lecture]

Everything is autoimmune until proven otherwise - yet every autoimmune condition is infectious until proven otherwise

Yehuda Shoenfeld

Tel-Aviv University, Israel

Abstract not received.

\section{[17.30-17.50]}

Peroxynitrite induced modification of human DNA: Implications in etiopathogenesis of SLE Dr. Moinuddin

Department of Biochemistry, J.N. Medical College, Faculty of Medicine, Aligarh Muslim University, Aligarh (U.P.), India

E-mail: moin_u@rediffmail.com

Abstract: Systemic Lupus Erythematosus (SLE) is a multisystem autoimmune disease involving both humarol and cellular aspects of innate and acquired immune system. The disease is characterized by the production of a variety of autoantibodies against nuclear, cytoplasmic and cell surface antigens. The cellular and molecular mechanisms that are responsible for the production of anti-nuclear antibodies in this disease and the way in which these antibodies participate in tissue destruction remain highly debated. Studies on IgG class switching and somatic mutations in $\mathrm{VH}$ regions of anti-DNA antibodies suggest that potentially pathogenic anti-DNA antibodies are the products of T-cell dependent autoantigen-driven immune responses. The primary autoantigen is believed to be DNA modified or complexed in some form rather than the naked analogue. Modification in DNA can take place through interaction with various radicals, radiations, other molecules or chemicals leading to Bhelix conformational alteration or generation of single stranded regions. One such species is peroxynitrite radical which causes oxidative DNA damage. In our study we have characterized peroxynitrite radical damaged human DNA through spectroscopic and fluorometric analysis, nuclease S1 studies, alkaline Agarose assay and thermal denaturation profile assays. The modified DNA was found to be highly unstable having appreciably high percentage of SS regions with the damage to backbone and disruption in base stacking. Binding of naturally occurring SLE autoantibodies to the native and modified DNA shows higher recognition of the modified conformer by the SLE autoantibodies in direct binding and completion ELISA experiments as well as in gel-retardation assays clearly indicationg that the modified DNA present better epitopes for the SLE autoantibodies. Role of peroxynitrite radical in the induction of SLE anti-DNA autoantibodies has been discussed.

[17.50-18.20]

Generation of anti-CD44-rheumatoid arthritis-specific monoclonal antibodies

David Naor and Itshak Golan

Lautenberg Center for General and Tumor

Immunology, The Hebrew University, Hadassah

Medical School, P.O.B. 12272, Jerusalem 91120 ,

Israel

Abstract: A selective targeting of cells engaged in pathological activities is a major challenge for medical research. We generated monoclonal antibodies (mAbs) that exclusively bind, at concentrations ranging from 0.2 to $100 \mathrm{ug} / \mathrm{ml}$, to a CD44 mutated variant (designated CD44vRA) expressed on synovial fluid cells from joints of rheumatoid arthritis (RA) patients. The same mAbs cross-react with keratinocytes expressing the wild type of CD44vRA (CD44v3-v10) only at a relatively high concentration $(200 \mathrm{ug} / \mathrm{ml})$. Sequence analysis of CD44vRA cDNA revealed, in 23 
out of 30 RA patients, an extra intron-derived trinucleotide, CAG, which allows translation of extra alanine (Nedvetzki et al., Journal of Clinical Investigation, $111,1211-1220,2003)$, resulting in a configurational change in the cell surface CD44 of RA synovial fluid cells and consequently an ability to produce diseasespecific antibodies. The anti-CD44vRA mAb (designated F8:33), interfered with the transwell cell migration of RA synovial fluid cells, but not with that of normal keratinocytes or synovial fluid cells of osteoarthritis patients, proving that F8:33 is a bioactive and se- lective mAb. Using animal models of autoimmunity, the pathological activities in collagen-induced arthritis of DBA/1 mice (J. Autoimmunity, 13, 39-47, 1999) and spontaneous type 1 diabetes of NOD mice (PNAS, 97, 285-290, 2000) were reduced following injection of commercial anti-CD44 mAb, even when the antibodies were administered after the disease onset. The mechanism of the CD44 targeting in the context of cell migration and Th1-Th2 cytokine balance is now under investigation. 